\title{
Vertical integration of an electro-absorption modulator within a VCSEL device
}

\author{
Ludovic Marigo-Lombart ${ }^{1,2}$, Alexandre Arnoult ${ }^{1}$, Christophe Viallon ${ }^{1}$, Stéphane Calvez ${ }^{1}$, \\ Aurélie Lecestre ${ }^{1}$, Benjamin Reig ${ }^{1}$, Alexandre Rumeau ${ }^{1}$, Hugo Thienpont ${ }^{2}$, Krassimir Panajotov ${ }^{2}$, and \\ Guilhem Almuneau ${ }^{1}$ \\ ${ }^{1}$ LAAS - CNRS, Université de Toulouse, CNRS, F-31400 France \\ ${ }^{2}$ Department of Applied Physics and Photonics (TW-TONA), Vrije Universiteit Brussel, Pleinlaan 2, B-1050 \\ Brussels, Belgium \\ E-mail: lmarigol@laas.fr
}

\begin{abstract}
The huge increase of datacom traffic requires laser sources of ever-widening modulation bandwidth. Vertical-Cavity Surface-Emitting Lasers (VCSEL) are strategically relevant given that their wide use for short communication links such as in datacenters and are, in light of recent developments, good candidates to address such demands. We propose to increase the modulation bandwidth by vertically integrating a continuous-wave VCSEL with a high-speed electroabsorption modulator (EAM-VCSEL). We will present our studies on the electrical and optical designs, on the development of optimized fabrication steps and on the microwave characterizations leading to the realization of integrated EAM-VCSEL devices.
\end{abstract}

Keywords: High-frequency modulation, Vertical Cavity Lasers, Electro-absorption modulator, Self-aligned technological processing, BCB planarization.

\section{INTRODUCTION}

Vertical Cavity Surface Emitting Lasers (VCSEL) offer several advantages compared to edge-emitting lasers, such as low drive current, low power consumption, high conversion efficiency, small size and dense integration capabilities within two-dimensional arrays enabling to fabricate in a single process a huge chip number per wafer. Also, VCSELs can achieve high-speed data transmission capabilities over short distance ranges, as well as a high beam quality for easy coupling to optical fibers.

For all these reasons VCSELs are today widely deployed in many applications including optical mice, atomic clocks, shortlink optical networks, and optical links in datacenters and supercomputers. Since recently, the VCSEL market has widely diversified with emerging applications such as 3-D imaging systems (Project Tango: Princeton Optronics \& Google [1]), imaging and gesture recognition (Intel RealSense project), surgery, displays, sensing, printing, high power for metallurgy industry, miniature embedded atomic clock, industrial optical sensors (Philips).

In order to improve the bandwidth of the VCSEL devices, and establish their applications potentialities, very different approaches and strategies have been applied since the 1990's. The most convenient solution being the direct modulation of the VCSEL device, and this approach has been driven on to its current limit above $30 \mathrm{GHz}$ enabling data links near $70 \mathrm{~Gb} / \mathrm{s}$ [2][3].

The proposal of a dual-cavity VCSEL is another interesting solution for increasing the modulation bandwidth. As described by Van Eisden in 2008 [4] this method avoids the limits of carrier dynamics on the intrinsic modulation and so will permit higher bandwidth. The idea of an external modulation is also advantageous to increase the device lifetime which is degraded by the high drive current. This device concept, demonstrated in 2012 [5], comprises of an Electro-Optic Modulator on top of the VCSEL with both cavities having similar resonance wavelengths. Because of its ability to change the photon density by varying the gain or absorption in one cavity, the Composite-Resonator VCSEL is another good candidate for high speed modulation as described by Choquette [6].

We have seen the different approaches investigated to improve VCSEL modulation with vertically integrated modulators or coupled cavities, but other alternative approaches like the transverse coupled cavity [7] and the combination of a transistor and a VCSEL [8] have also been studied.

Finally, another simple mean to increase bandwidth for data communication is the design of VCSEL arrays as done by Larsson et al. [9].

In this paper we propose to develop a VCSEL with an integrated Electro-Absorption Modulator (EAM_VCSEL), enabling to overcome the actual physical limits imposed by todays VCSEL technology and increase significantly the modulation bandwidth of a single device, in order to meet the upcoming 100G Ethernet standard. Our EAM-VCSEL structure is schematically shown in Figure 1. It consists of an N-doped GaAs substrate on which the structure is grown. From bottom to top, the structure features one N-doped DBR, intrinsic multiple quantum wells, an AlGaAs layer for lateral oxidation for electrical and optical confinement, a P-doped DBR - which is shared with the modulator-, multiple quantum wells for absorption and a top N-doped DBR. The 
operation principal of this EAM-VCSEL device is splitted into two main functionalities: a VCSEL light source delivering a continuous laser emission with controlled properties (power, efficiency, modal properties), and a electro-absoprtion modulator carefully designed in order to efficiently modulated the transmission of the output laser emission. The low coupling between the two optical cavities will enable to avoid the undesirable interactions between them.

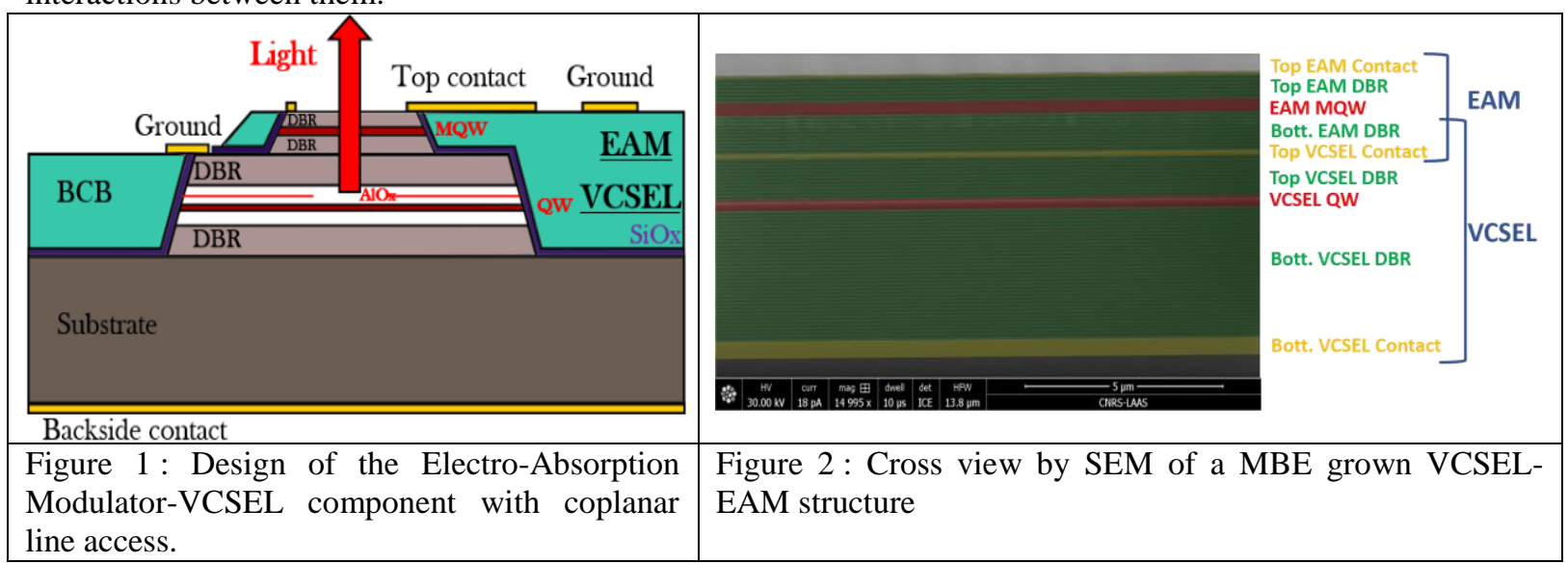

\section{TECHNOLOGICAL DEVELOPMENTS}

\subsection{Molecular beam epitaxy of the EAM and EAM-VCSEL structures}

Because of the extremely high number of layers in our EAM-VCSEL structure, the growth sequence is very complicated and requires a huge upstream work for the calibration of layer thicknesses and the alloys compositions. The growth of the different vertical structures has been realized by Molecular Beam Epitaxy using a Riber 412 reactor, equipped with 2 duplicated effusion cells for $\mathrm{Ga}, \mathrm{Al}, \mathrm{In}$ and $\mathrm{As}$ elements, and for doping cells: $\mathrm{Si}$ and $\mathrm{CBr}_{4}$. The growth conditions such as wafer temperature and growth rates are precisely and real-time in-situ controlled respectively by a bandgap optical measurement with the KSA BandiT sytem, and by optical reflectometry with a home-made system.

High resolution X-ray diffraction, normal reflectivity, and high resolution Scanning Electron Microscopy are used as post-growth ex-situ characterization tools to retrieve the compositions and thicknesses of individual layers and compare them to the designed structure.

Thanks to this rigorous method, five different EAM and EAM-VCSEL structures have been successfully grown, for setting up a short design of experiments with different types of vertical structures aiming to check:

-The impact of the substrate doping on the high frequency properties,

-The impact of the DBR doping on the drive voltage and $\mathrm{R}$ and $\mathrm{C}$ parameters,

- The influence of the wavelength detuning between EAM and VCSEL's cavities.

\subsection{Self aligned technological process for the EAM fabrication}

As presented on Figure 1, the full fabrication process flow for the realization of the EAM-VCSEL device requires three contact pads and two mesas in order to address independently the VCSEL and the modulator devices. Moreover, these different patternings must be strictly aligned in order to optimize the VCSEL performances and the modulator efficiency.

Since the numerous photolithography steps with subsequent perfect mask alignments are extremely challenging, we proposed to implement a self-align process for different process steps. The self-align fabrication process will drastically release the constraints on the patterns alignments, but also will enable to simplify and thus make more reliable the process flow by minimising the total number of photolithography steps. The original self aligned process flow that we developed is described in [10] and has been successfully applied to air-post and oxide laterally confined VCSELs as shown in Figure 3, thus proving its applicability for the both parts of the EAMVCSEL device.

This innovative technological solution allows to perform consecutively, from a single lithographic step, the mesa dry etching, the surface passivation with an open emission window, and the annular top contact. Thus, it is of high interest since it drastically simplifies and improves the repeatability of the fabrication of the VCSEL / modulator devices, but additionally its application can be advantageously transferred to different kind of photonic and microelectronic devices. 


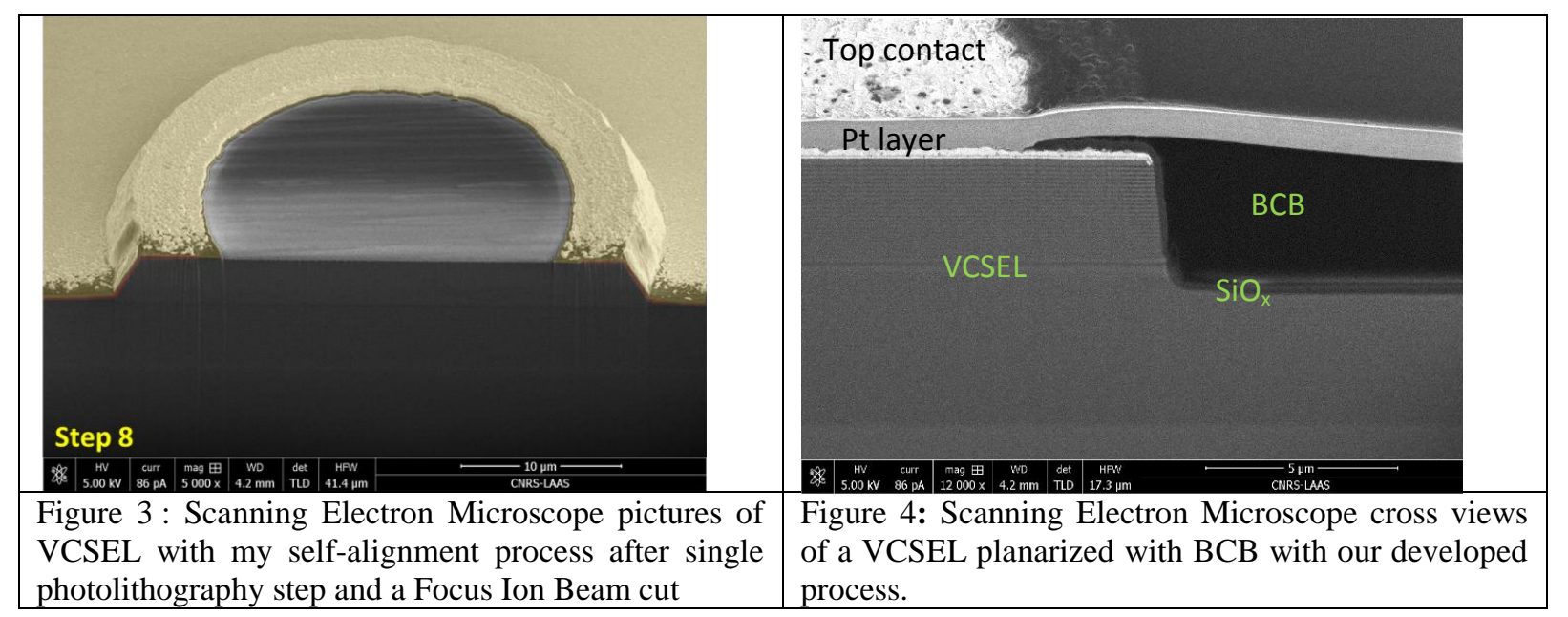

\subsection{BCB planarization process using pressing technique}

In order to limit the capacitance between contact pads in the final structure, thick insulating layer is deposited around the etched mesas. The BCB (bisbenzocyclobutane) has been extensively used in microwave and photonics applications because of its good insulating properties at very high frequencies up to the $100 \mathrm{GHz}$ range. This polymer has also the advantage to be easily applied by spin-coating on the wafer surface, but nevertheless its low viscosity make the planarization highly difficult. This issue is especially problematic in our case of the EAM-VCSEL structure since BCB coating must be applied to profile height of several microns. We have then developed an adapted process to improve the BCB polyimide planarization in order to ensure a perfect planarization on any surface profiles [11]. The feasibility of this process has been qualified on a VCSEL (Figure 4). Static and high frequency measurements on these devices have to be done to check that we did not affect its optical properties while improving its electrical properties.

\section{DEVICES CHARACTERIZATION}

\subsection{Static characterization of the Electro-Absorption Modulator}

We have fabricated stand-alone EAM structures to measure the reflectivity of the modulator as a function of the applied DC voltage. The different samples have been measured on a homemade dedicated reflectivity measurement bench. Results are shown on Figure 5.

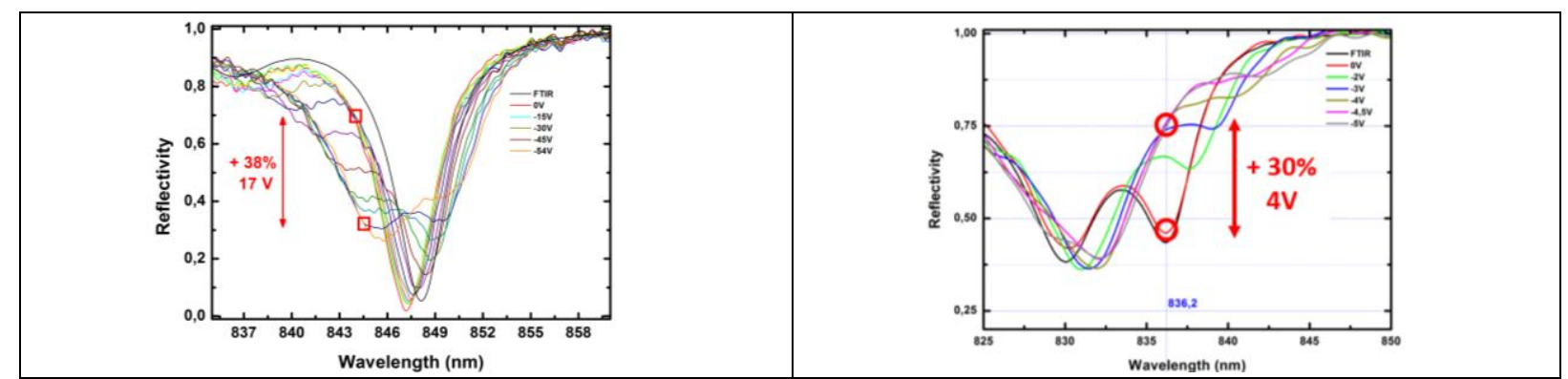

Figure 5 : Reflectivity measurement function of applied voltage, on the EAM with undoped DBR (on the left), on the EAM with doped DBR (on the right).

In the EAM structure for which the DBRs are undoped, so implying a higher reverse applied voltage (i.e. about $17 \mathrm{~V}), 38 \%$ of modulation depth has been achieved. Since with the other EAM structure with doped DBRs, only $4 \mathrm{~V}$ are necessary to reach this $30 \%$ modulation ratio. These results are on a par with the state-of-the art as reviewed by Yan et al. [12].

\subsection{High frequency characterization of BCB films, and contact pad microwave design}

The design and the characterization of the electrical injection play a key role in order to decrease as much as possible the parasitic losses through the (BCB) insulating layer and to achieve high-frequency operation.

We designed, with High Frequency System Simulator (HFSS), and fabricated coplanar access line in order to realize the high frequency characterization of the BCB layer upon different types GaAs substrates up to 110 GHz. Open and short design lead to the validation of the simulation and transmission design leads to the extraction, with a T-model in Advanced Design System (ADS), to the R, L and C parameters of the injection pad. We tested the coplanar lines access on three different wafers, a Not Intentionally Doped (NID) substrate, a P-substrate and a N-substrate to have a first feedback on our simulation. Due to the uncertainty on the substrate 
doping and on the BCB permittivity given by the supplier we decided to use the method developed by Ghannam et al [13]. We extracted a first value of 2.65. However, $\mathrm{N}$ and $\mathrm{P}$ substrates have the drawback to increase the leakage and Foucault currents; while in the case of the NID substrate we should have to add a third contact at the foot of the VCSEL which would considerably complicate the process. We decided to test microstrip line access in our ADS simulation to decrease the losses at high frequency and obtained very encouraging results.

\section{CONCLUSIONS}

In conclusion, we propose a novel approach of vertical integration of a VCSEL and a high speed electroabsorption modulator in order to avoid physical limitation due to photon-carrier dynamics in directly modulated VCSELs. We present different technological developments such as self-aligned VCSEL process and BCB planarization. BCB was electrically characterized and our high frequency design improved to decrease losses in the substrate. We present the fabrication by MBE of EAM and EAM-VCSEL structures. First optical characterizations are very encouraging since they fit with the state-of-the-art performances.

\section{ACKNOWLEDGEMENTS}

The authors gratefully acknowledge the technological support of RENATECH (French Network of Technology Platforms) within LAAS-CNRS cleanroom infrastructure and the financial support by the Methusalem foundation, Belgium.

\section{REFERENCES}

[1] https://www.photonics.com/Article.aspx?AID=57110

[2] Kuchta, D.M. and Rylyakov, A.V. and Schow, C.L. and Proesel, J.E. and Baks, C. and Westbergh, P. and Gustavsson, J.S. and Larsson, A., "64Gb/s transmission over 57m MMF using an NRZ modulated 850nm VCSEL", Optical Fiber Communications Conference and Exhibition (OFC), 2014.

[3] Kuchta, D.M. and Rylyakov, A.V. and Doany, F.E. and Schow, C.L. and Proesel, J.E. and Baks, C.W. and Westbergh, P. and Gustavsson, J.S. and Larsson, A., "A 71-Gb/s NRZ Modulated 850-nm VCSEL-Based Optical Link”, Photonics Technology Letters, vol. 27, no. 6, p. 577-580, Mar. 2015.

[4] Van Eisden, J. and Yakimov, M. and Tokranov, V. and Varanasi, M. and Mohammed, E.M. and Young, I.A. and Oktyabrsky, S.R, "Optically Decoupled Loss Modulation in a Duo-Cavity VCSEL", Photonics Technology Letters, vol. 20, no. 1, p. 42-44, Jan. 2008.

[5] Tim David Germann and Werner Hofmann and Alexey M. Nadtochiy and Jan-Hindrik Schulze and Alex Mutig and André Strittmatter and Dieter Bimberg, "Electro-optical resonance modulation of vertical-cavity surface-emitting lasers", Opt. Express, vol. 20, no. 5, p. 5099-5107, Feb. 2012

[6] Chen, C. and Leisher, P.O. and Grasso, D.M. and Long, C. and Choquette, K.D., "High-speed electroabsorption modulation of composite-resonator vertical-cavity lasers",Optoelectronics, IET, vol. 3, no. 2, p. 93-99, Apr. 2009.

[7] Hamed Dalir and Fumio Koyama, "40 Gbps modulation of transverse coupled cavity VCSEL with pushpull modulation scheme", Applied Physics Express, vol. 7, no. 9, 2014.

[8] M. Nadeem Akram and Y. Xiang and X. Yu and Thomas Zabel and Mattias Hammar, "Influence of baseregion thickness on the performance of Pnp transistor-VCSEL", Opt. Express, vol. 22, no. 22, p. 2739827414, Nov. 2014.

[9] Westbergh, P. and Gustavsson, J.S. and Larsson, A., "VCSEL Arrays for Multicore Fiber Interconnects With an Aggregate Capacity of 240 Gb/s", Photonics Technology Letters, vol. 27, no. 3, p. 296-299, Feb. 2015

[10] L. Marigo-Lombart, A. Arnoult, L. Mazenq, P. Dubreuil, B. Reig, N. Mauran, H. Thienpont, K. Panajotov and G. Almuneau, "Single lithography-step self-aligned fabrication process for Vertical-Cavity SurfaceEmitting Lasers", Mater. Sci. Semicond. Process., vol. 61, p. 35-38, April 2017

[11] L. Marigo-Lombart, J-B. Doucet, A. Lecestre, B. Reig, B. Rousset, H. Thienpont, K. Panajotov and G. Almuneau, "Self-aligned BCB planarization mathoed for high-frequency signal injection in a VCSEL with an integrated modulator", Proc. SPIE 9892, April 2016

[12] Yan, R.H. and Simes, R.J. and Coldren, L.A., "Surface-Normal Electroabsorption Reflection Modulators Using Asymmetric Fabry-Perot Structures”, J. of Quantum Elec., vol. 27, no. 7, July 1991

[13] A. Ghannam, C. Viallon, D. Bourrier and T. Parra, "Dielectric microwave characterization of the SU-8 thick resin used in an above IC process," 2009 European Microwave Conference (EuMC), Rome, 2009, pp. 1041-1044 